\title{
On the formation of rings-on-a- string conformations in a single polyelectrolyte chain: A possible scenario
}

\section{$\operatorname{AUTHOR}(\mathrm{S}):$}

Sakaue, Takahiro; Yoshikawa, Kenichi

\section{CITATION:}

Sakaue, Takahiro ... [et al]. On the formation of rings-on-a-string conformations in a single polyelectrolyte chain: A possible scenario. Journal of Chemical Physics 2006, 125(7): 074904.

\section{ISSUE DATE:}

2006-08-21

URL:

http://hdl.handle.net/2433/45687

\section{RIGHT:}

Copyright (2006) American Institute of Physics. This article may be downloaded for personal use only. Any other use requires prior permission of the author and the American Institute of Physics. 


\title{
On the formation of rings-on-a-string conformations in a single polyelectrolyte chain: A possible scenario
}

\author{
Takahiro Sakaue a) and Kenichi Yoshikawa \\ Department of Physics, Graduate School of Science, Kyoto University, Kyoto 606-8502, Japan, \\ and Spatio-Temporal Order Project, ICORP, JST, Kyoto 606-8502, Japan
}

(Received 30 May 2006; accepted 6 July 2006; published online 18 August 2006)

\begin{abstract}
Recent single-molecular observations have revealed that a single giant DNA molecule assumes (micro) phase separated structures upon the addition of condensing agents. Electron and atomic force microscopy have clearly shown the coexistence of ordered tori and disordered coil structures within a single DNA molecule. Motivated by these experimental findings, we theoretically investigated the collapse transition of a single polyelectrolyte chain driven by the addition of condensing guest molecules. We found that the transition behavior critically depends on the degree of the surviving charge inside the torus. When the torus is charged, even slightly, "rings-on-a-string" structures are expected for a sufficiently long chain, owing to the combinational entropy of segment state distribution along the chain and the unique property of the stability of charged torus. (C) 2006 American Institute of Physics. [DOI: 10.1063/1.2244555]
\end{abstract}

\section{INTRODUCTION}

Within living cells, long DNA molecules exist in a tightly packed compact state. It is expected that the function of DNA is highly correlated to its structure and manner of packing. Therefore, understanding the manner of DNA folding is a necessary step to unveil the mechanism that underlies many fundamental biological processes, such as DNA replication and transcription. Accordingly, the issue of DNA compaction has promoted a lot of theoretical and experimental studies. ${ }^{1-3}$ In very dilute solution, individual DNA molecules are collapsed by the addition of various kinds of condensing agents. This conformational transformation has been shown to be a discrete transition at the level of individual chains, ${ }^{4}$ in contrast to the classical scenario, a gradual shrinking through the $\theta$ point. ${ }^{1}$ The collapsed structure is not a liquidlike spherical globule but rather a crystalline structure with local orientational order, and its morphology is typically a torus. Such features are expected to be a consequence of the chain stiffness. In fact, computer simulation studies have shown that a stiff or semiflexible chain folds from a swollen coil into a crystalline torus structure discontinuously. ${ }^{5-8}$

The recent development of single-molecular observation has revealed that an all-or-none discrete transition is not the only type of DNA folding. Instead, DNA molecules develop structural heterogeneities, where collapsed parts and coil parts coexist within a single chain under certain conditions. These partially collapsed states are stable within a certain range of condensing agent concentration, and the further addition of condensing agent leads to complete collapse. ${ }^{9-11}$ This observation has promoted several theoretical attempts. ${ }^{11-14}$ At certain conditions, experiments have revealed that the partially collapsed parts take an ordered torus

\footnotetext{
a) Also at Yukawa Institute for Theoretical Physics, Kyoto University. Electronic mail: sakaue@scphys.kyoto-u.ac.jp
}

structure, which are interconnected by coil parts. ${ }^{15,16}$ Hence, a single DNA chain takes a "rings-on-a-string" conformation, as shown in Fig. 1.

This rings-on-a-string structure is characterized by the coexistence of ordered domains (torus) and disordered domains (coil), thus, regarded as microphase segregation within a single chain. Since the generation of an ordered folded structure from a semiflexible chain can be considered a kind of crystal growth, the appearance of such intrachain segregated structures is somewhat counterintuitive. In fact, in the simulation of the folding of a single semiflexible chain, where the process of torus nucleation and growth is clearly observed, a partially folded structure with a growing torus is only transient, and never stable. ${ }^{17}$

One may suppose that this phenomenon is caused by Rayleigh instability; a single droplet becomes unstable upon the accumulation of the charge due to long-range nature of the electrostatic interaction. ${ }^{17}$ A polyelectrolyte chain in poor solvent, thus, may assume the pearl-necklace structure, in which multiple small globules are interconnected by strings. ${ }^{18-20}$ However, it is not immediately obvious that this mechanism is responsible for the rings-on-a-string structures observed for DNA in solution with moderate concentration of the monovalent salt. In fact, a simple energetic consideration suggests the following unique characteristic of the charged torus. ${ }^{21}$ At a given segment density, a torus is characterized by two characteristic radii of curvature, i.e., ring radius and ring thickness, therefore, possesses larger degree of freedom than a spherical globule, which is solely characterized by the radius, or equivalently, by the segment number inside it. This additional freedom provides an escape pathway for the torus to grow without accumulating the electrostatic self-energy, i.e., unlike a spherical globule, a torus does not necessarily split upon the charging. As an alternative 
(a)

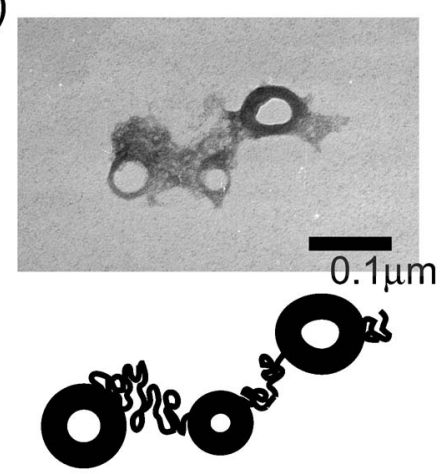

(b)

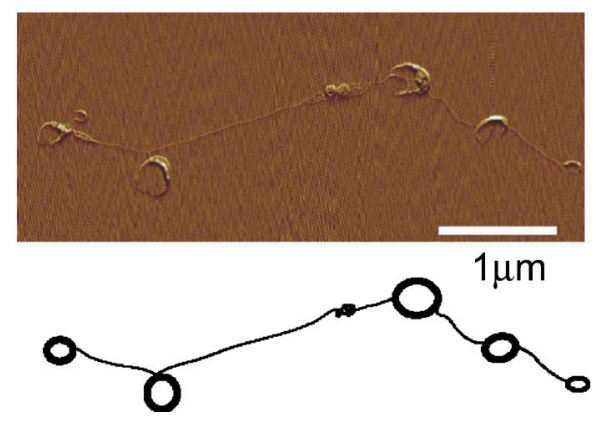

FIG. 1. (Color online) Examples of a single DNA molecule in rings-on-astring conformations and their schematic pictures. (a) A transmission electron micrograph and (b) an atomic force microscopic image of a single T4 DNA molecule (contour length: $57 \mu \mathrm{m}$ ), collapsed by the addition of (a) a quaternary diammonium compound and (b) a gemini surfactant. (a) and (b) are adapted from Refs. 15 and 16, respectively. Reprinted with permission from Ref. 15. Copyright (2003) American Chemical Society.

reason, one may also suppose that such segregations are caused due to heterogeneity in the base pair sequence of DNA.

In Ref. 16, Miyazawa et al. reported the experimental evidence on the rings-on-a-string structure in DNA together with a rough theoretical expectation base on Ref. 12. In the present article, we present theoretical ideas in more details, and address the question of whether a charged semiflexible homopolymer takes rings-on-a-string structures under thermal equilibrium.

\section{THEORY}

To investigate the collapsing behavior of a DNA molecule, we consider a homopolymer with contour length $L$, Kuhn length $l$ (thus, the total number of statistically independent segments is $\left.N_{0}=L / l\right)$, and line charge density $\rho$, which is embedded in a volume $\Omega$. Throughout this article, all lengths and energies are measured in units of segment diameter and thermal energy $k_{B} T$, respectively. The solution contains a moderate concentration of monovalent salts such as $\mathrm{NaCl}$, and additional guest molecules as a condensing agent with valency $z$, whose number concentration is denoted as $c_{g}$. For simplicity, we consider the case of $z^{-1}<\rho l_{B}<1\left(l_{B}\right.$ is the Bjerrum length), and thus the monovalent ions simply provide conventional linear screening with a decay length of $r_{D}$, comparable to the scale of a segment diameter.
We introduce a constrained free energy for the rings-ona-string structure with alternating $n_{t}$ tori and $n_{c}$ coil parts as

$$
F\left(n_{t},\left\{N_{i}\right\} ; n_{c},\left\{N_{j}\right\}\right)=\sum_{i=1}^{n_{t}} F_{t}\left(N_{i}\right)+\sum_{j=1}^{n_{c}} F_{c}\left(N_{j}\right)+F_{\text {out }},
$$

where $\left\{N_{i}\right\}$ and $\left\{N_{j}\right\}$ designate $i$ and $j$ dimensional vectors, respectively. $F_{t}\left(N_{i}\right), F_{c}\left(N_{j}\right)$, and $F_{\text {out }}$ are the free energies of a torus with $N_{i}$ segments, a coil with $N_{j}$ segments, and the outer solution, respectively. Note that for $n_{t}>1, n_{c}=n_{t}-1, n_{t}$, or $n_{t}+1$, while for $n_{t}=1, n_{c}=1$ or 2 , depending on the states of the chain ends. ${ }^{29}$ The interactions between different coils and/or tori are negligibly small under the present condition $\left(r_{D} \sim 1\right)$.

A torus, inside which segments are densely packed in a parallel arrangement, is generated due to the attractive interaction caused by the condensation of guest molecules. Although the fine structure inside the torus, i.e., how many guest molecules are condensed and how they are distributed inside torus, depends on several experimental parameters (type of condensing agents, salt concentration, etc.), it is possible to discuss torus stability at a phenomenological level. We consider a torus with $N_{i}$ segments, inside which $M_{i}$ guest molecules are condensed. The free energy of a torus with two characteristic radii of curvature $R_{i}$ (ring radius) and $r_{i}$ (ring thickness) can be decomposed as ${ }^{21}$

$$
F_{t}(N, R, r)=F_{t}^{\mathrm{vol}}+F_{t}^{\mathrm{sur}}+F_{t}^{\mathrm{bend}}+F_{t}^{\mathrm{elec}}+F_{t}^{\mathrm{conf}}
$$

where we omit the subscript representing the $i$ th torus to avoid vexatious notations. The first two terms are the stabilization energy (proportional to torus volume), $F_{t}^{\mathrm{vol}} \simeq \epsilon r^{2} R$, and surface energy, $F_{t}^{\text {sur }} \simeq \gamma r R$, where $\epsilon(<0)$ and $\gamma$ are the cohesive energy density and surface tension, respectively. The third term is associated with bending to form a torus, $F_{t}^{\text {bend }} \simeq \kappa N l / R^{2}$ with bending modulus $\kappa=l / 2$. The fourth term represents the electrostatic self-energy of a torus, which can be approximated by that of a cylinder with radius $r$, length $R, F_{t}^{\text {elec }} \simeq l_{B}\left[(\rho N l-z M)^{2} / R\right] \ln \left[\left(r+r_{D}\right) / r\right]$. The last represents the possible contribution of the configurational entropy of guest molecules inside the torus. The precise form of this term does not affect the qualitative picture in the present paper, therefore, for simplicity, we adopt the ideal gas approximation in the following calculations.

To proceed further without detailed knowledge on the internal structure of the torus, we adopt the assumption that the degree of charge neutralization inside the torus, $\alpha \equiv(1-z M / \rho N l)$ is a given parameter that depends on the nature of the guest molecules and their interaction with polyelectrolytes. Note that the surviving charge inside the torus is expected to be probable if the collapse is stabilized by not only electrostatic but also nonelectrostatic (hydrophobic, etc.) forces. Using the relationship $N l a^{2} \simeq r^{2} R$, which means that the segments inside the torus are most densely packed, the expression for the free energy of a torus with given $\alpha$, Eq. (2), is reduced to a function of $r$. The optimum size of a torus is derived via variational calculation on $r$.

For the remaining free energy terms, we adopt an argument that follows a type of counterion condensation theory. ${ }^{22,23}$ Owing to the balance between the electrostatic 
energy of a coil and the translational freedom of guest molecules, some fraction of guest molecules are condensed on the chain. The free energy of a coil part is expressed as the sum of the electrostatic energy of a string with renormalized charge and the configurational entropy of condensed guest molecules and the conformational entropy of the coil,

$$
F_{c}=F_{c}^{\mathrm{elec}}+F_{c}^{\mathrm{conf}}+F_{c}^{\mathrm{chain}} .
$$

These terms can be expressed in linear form with respect to $N_{j} . \quad F_{c}^{\mathrm{elec}}\left(N_{j}\right) \simeq l_{B} l N_{j}(\rho-m)^{2} \ln \left(1+2 r_{D}\right)$, $F_{c}^{\mathrm{conf}}\left(N_{j}\right) \simeq l N_{j}[m \ln (m z / \rho)+((\rho / z)-m) \ln (1-m z / \rho)], \quad F_{c}^{\text {coil }}$ $\left(N_{j}\right) \simeq-\phi N_{j}$, where $m$ is the number of condensed guest molecules on the chain per unit length, and $\phi$ is the constant order of unity associated with the chain conformation.

The free energy of the outer solution mainly arises from the translational entropy of free guest molecules, $F_{\text {out }}$ $\simeq M_{\text {free }} \ln \left(M_{\text {free }} / \Omega\right)$, where $M_{\text {free }}=c_{g} \Omega-\sum_{i=1}^{n_{t}} M_{i}-m l \sum_{j=1}^{n_{c}} N_{j}$ is the number of free guest molecules. The degree of counterion condensation is determined via variational calculation on $m$.

After substituting the optimum free energy of each torus and coil into Eq. (1), we obtain the optimum free energy of the polyelectrolyte in the rings-on-a-string conformation with preassigned segment distribution characterized by $n_{t}$ tori and $n_{c}$ coils, which is denoted as $F^{*}\left(n_{t},\left\{N_{i}\right\} ; n_{c},\left\{N_{j}\right\}\right)$. To seek the stable conformations, it is important to notice that the number of statistically independent segments in a polymer chain is, even though quite large, much smaller than typical thermodynamic systems. Therefore, the segment distribution should be exposed to a rather strong thermal fluctuation. Since the quantity of our interest is the number of tori within a single polyelectrolyte, which is directly measured from microscopic observation, we introduce partition functions corresponding to specific states characterized by $n_{t}$ and/or $n_{c}$, i.e., $Z^{\mathrm{I}}$ (coil), $Z_{n_{t}}^{\mathrm{II}}$ (rings-on-a-string with $n_{t}$

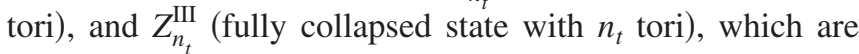
calculated with conservation of the segment number, $\Sigma_{i} N_{i}$ $+\sum_{j} N_{j}=N_{0}$

$$
\begin{aligned}
& Z^{\mathrm{I}}=\exp \left\{-F^{*}\left(0 ; 1,\left\{N_{0}\right\}\right)\right\} \\
& Z^{\mathrm{II}}=\sum_{n_{t}} Z_{n_{t}}^{\mathrm{II}}=\sum_{n_{t}} \sum_{n_{c}} \sum_{\left\{N_{i}\right\}} \sum_{\left\{N_{j}\right\}} \exp \left\{-F^{*}\left(n_{t},\left\{N_{i}\right\} ; n_{c},\left\{N_{j}\right\}\right)\right\}
\end{aligned}
$$

$$
Z^{\mathrm{III}}=\sum_{n_{t}} Z_{n_{t}}^{\mathrm{III}}=\sum_{n_{t}} \sum_{\left\{N_{i}\right\}} \exp \left\{-F^{*}\left(n_{t},\left\{N_{i}\right\} ; 0\right)\right\} .
$$

Note here that there is a possible occurrence of multiple tori as fully collapsed structures ${ }^{21}$ as is explicitly seen in Eq. (6). We numerically calculated $Z_{n_{t}}^{\mathrm{II}}$ and $Z_{n_{t}}^{\mathrm{III}}$ by enumerating all possible segment distributions. The grand partition function is

$$
\Xi=Z^{\mathrm{I}}+\sum_{n_{t}} Z_{n_{t}}^{\mathrm{II}} \exp \left(\mu_{t} n_{t}\right)+\sum_{n_{t}} Z_{n_{t}}^{\mathrm{III}} \exp \left(\mu_{t} n_{t}\right),
$$

where $\mu_{t}$ is the chemical potential of a torus. Note that $\mu_{t}=0$ reflects the fact that there is no conservation law for the generation and annihilation of the torus.
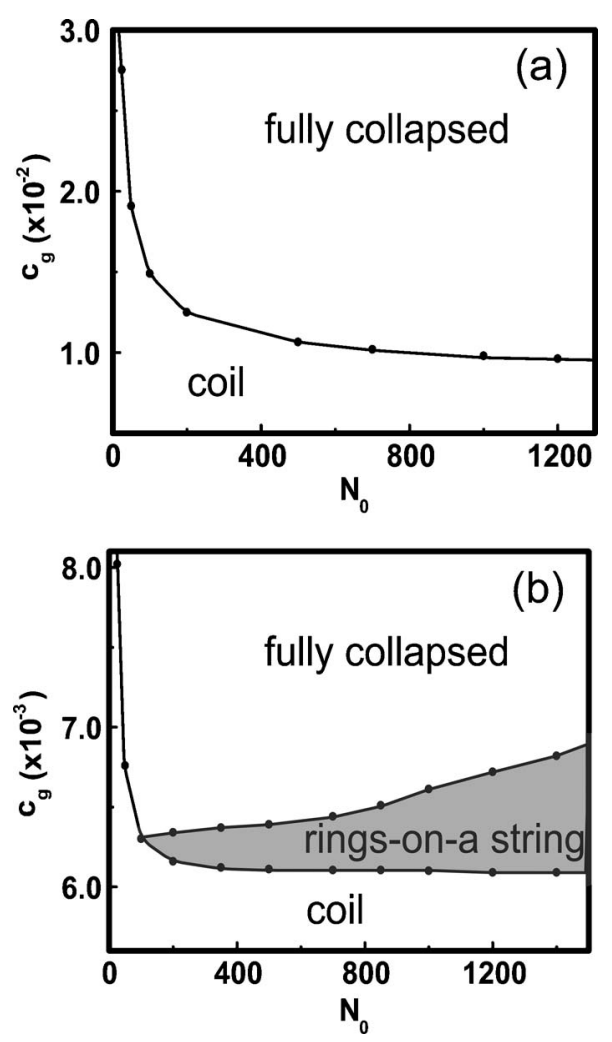

FIG. 2. State diagrams of a single polyelectrolyte chain as a function of the concentration of guest molecules $c_{g}$ and chain length $N_{0}$. (a) A torus is almost completely neutralized, $\alpha=10^{-4}$. (b) A torus is slightly charged due to the surviving excess charge, $\alpha=0.2$. In the region designated as rings on a string, rings-on-a-string conformations are most probable $\left(Z^{\mathrm{II}}>Z^{\mathrm{I}}, Z^{\mathrm{III}}\right)$.

\section{RESULTS AND DISCUSSIONS}

In Fig. 2, we exemplify a diagram of state for a single polyelectrolyte chain in the $\left(c_{g}-N_{0}\right)$ plane for two different cases; (a) the interior charge of the torus is almost neutralized by the condensation of guest molecules $\left(\alpha=10^{-4}\right)$, and (b) neutralization is incomplete, and thus the torus is slightly charged $(\alpha=0.2)$. Figures 3 and 4 show two quantities of interest around the transition region: (i) the average number of collapsed segment $\left\langle N_{t}\right\rangle$, and (ii) its fluctuation $\left\langle\Delta N_{t}^{2}\right\rangle^{1 / 2}$ for various chain lengths (bracket means the thermal average). In these calculations, we adopted the following parameters: $l_{B}=r_{D}=1, \rho=0.7, l=20, z=2, \epsilon=-0.4$, and $\gamma=0.15$. The system volume $\Omega$ is set so that the volume fraction of a chain $\left(\sim N_{0} l / \Omega\right)$ is $\sim 3 \times 10^{-4}$. Under these conditions, polyelectrolyte collapse does not occur until the total charge of condensing agents is much greater than that of a polyelectrolyte, with a ratio on the order of 100 .

In case (a), the transition is all-or-nothing between two states, coil and fully collapsed states. The size scaling of a torus is the same as that of a neutral chain. ${ }^{8,21,24-26}$ Reflecting the finite chain length effect, the transition concentration of guest molecules $c_{g}^{*}$ shows rather strong length dependence for a short chain region, where $c_{g}^{*}$ rapidly decreases with $N_{0}$.

On the other hand, qualitatively different behaviors are observed if there remains a slight surviving charge inside the torus [case (b)]. For short chains $\left(N_{0}<100\right)$, the transition is all-or-none type. Just like the case (a), the transition region becomes smaller with the increase in chain length. However, 

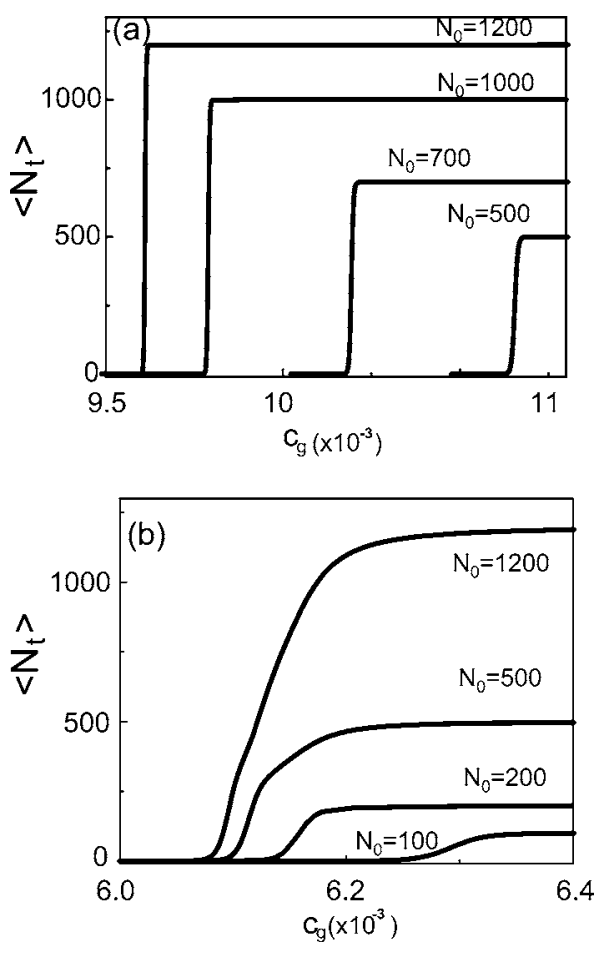

FIG. 3. Average number of segments inside torus state $\left\langle N_{t}\right\rangle$ around the collapse transition for (a) $\alpha=10^{-4}$ and (b) $\alpha=0.2$.

further increase in chain length converts the transition manner from the all-or-none to multistep folding. This is demonstrated by the appearance of the shoulder and/or secondary peak in the graph of $\left\langle\Delta N_{t}^{2}\right\rangle^{1 / 2}$ after the sharp first peak. Another signal for this multistep folding is recognized in the dependence of $\left\langle N_{t}\right\rangle$ on $c_{g}$, where clear inflectionlike points are seen for long chains. The first steep increase in $\left\langle N_{t}\right\rangle$ cor-
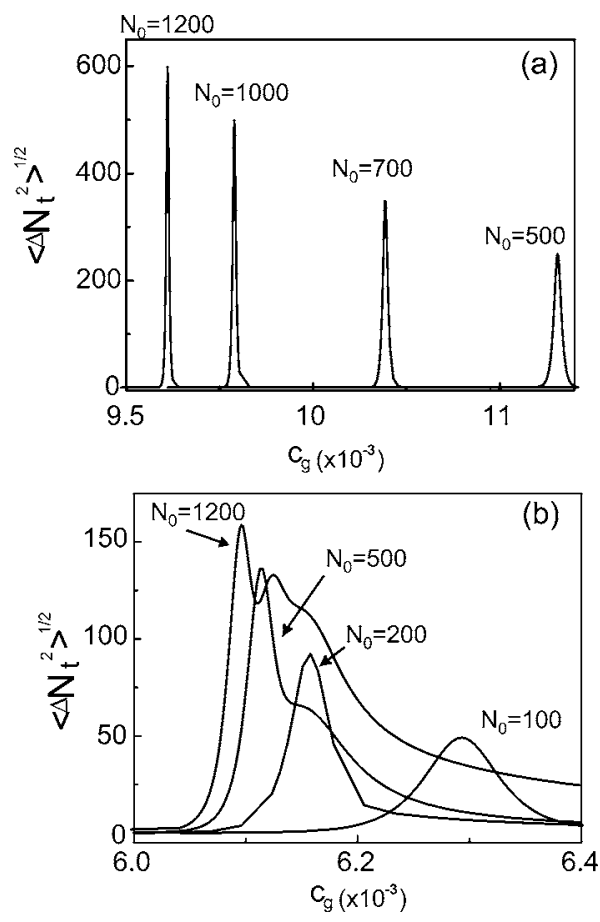

FIG. 4. Fluctuation of the number of segments inside torus state $\left\langle\Delta N_{t}^{2}\right\rangle^{1 / 2}$ around the collapse transition for (a) $\alpha=10^{-4}$ and (b) $\alpha=0.2$.
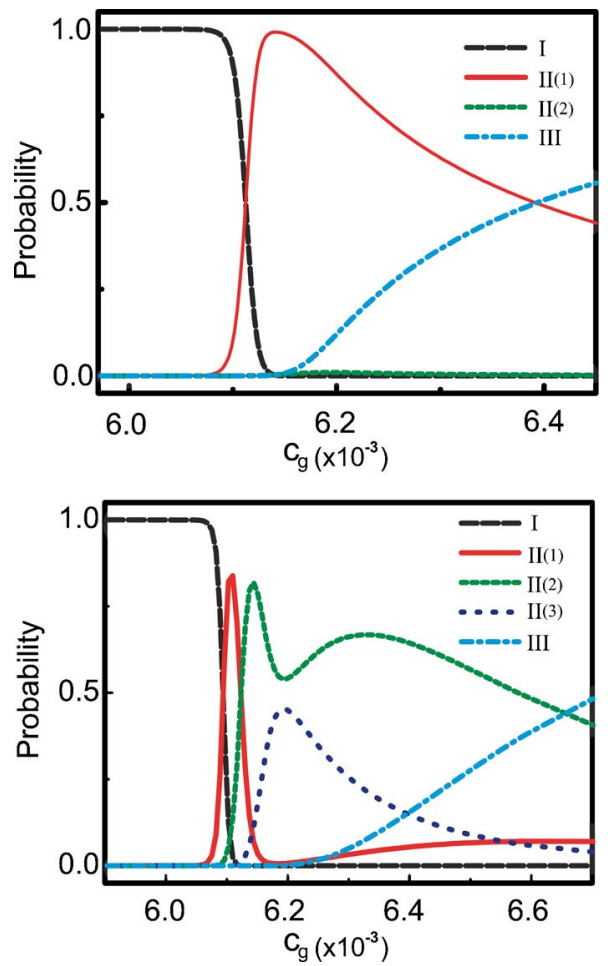

FIG. 5. (Color online) The probability distribution of polyelectrolyte structures depending on the concentration of guest molecules $c_{g}$ for a chain length of (top) $N_{0}=500$ and (bottom) $N_{0}=1200$. In both cases, the degree of the surviving charge inside the torus of $\alpha=0.2$. The most probable numbers of tori in the fully collapsed state are $n_{t}=1 \quad\left(N_{0}=500\right)$ and $n_{t}=2$ $\left(N_{0}=1200\right)$.

responds to the formation of the first torus, where the coil state and the intrachain segregated state with one torus coexist. It is seen that $c_{g}^{*}$, which is used for the guest molecule concentration at the onset of the collapse (first peak in $\left\langle N_{t}\right\rangle$ ) for the case of multistep folding, approaches the asymptotic value with the increase in chain length. The latter modest increase in $\left\langle N_{t}\right\rangle$ corresponds to the growth of the torus and/or the successive formation of a new torus. The region of the intrachain segregation becomes wider with the chain length. The detailed probability distributions depending on $c_{g}$ for $N_{0}=500$ and 1200 are shown in Fig. 5. In the former case, the intrachain segregation with one torus is most stable in some range of $c_{g}$, but two tori structure becomes never dominant, whereas in the latter case, the transition from one torus structure to two tori structure is seen, which is signaled in the second peak in $\left\langle\Delta N_{t}^{2}\right\rangle^{1 / 2}$. These results indicate that the longer chain has a potentiality to form rings-on-a-string structure with more tori, and its collapse transition is characterized by a sequence of transitions from one structure to the other with different number of tori.

These qualitatively different transition behaviors result from the difference in the excess interior charge of the torus $\alpha$. To see the effect of $\alpha$, it is instructive to consider the state with one torus and one coil part, and its corresponding free energy $F_{1}(N) \equiv F\left(n_{t}=1,\{N\} ; n_{c}=1,\left\{N_{0}-N\right\}\right)$. Generally $F_{1}(N)$ is decomposed as $F_{1}(N)=F_{1}^{\text {bulk }}+\Delta F_{1}$, where $F_{1}^{\text {bulk }}$ $=\Delta \mu N$ is the extensive part proportional to the segment number $(\Delta \mu$ is the difference between the segment chemical potentials in the coil and the torus states), and $\Delta F_{1}$ is the non- 


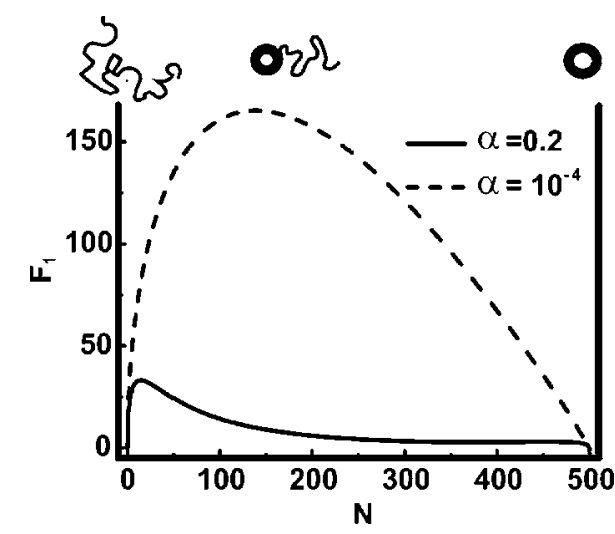

FIG. 6. Free energy profiles of a polyelectrolyte with one torus and one coil measured from the coil state as a function of the number of segments in the torus state. The chain length is $N_{0}=500$, and the degree of the surviving charge inside the torus is $\alpha=10^{-4}$ (dashed line, $c_{g} \simeq 6.14 \times 10^{-3}, \alpha=0.2$ (solid line, $c_{g} \simeq 1.07 \times 10^{-2}$ ).

extensive part that mostly arises from the surface and bending energy of the torus. In the case of a neutral chain, as a consequence of the competition between the surface and bending energies, the thickness of the torus scales as $r \sim N^{2 / 5}$. One can derive this by minimizing the relevant part of torus free energy $F_{t}^{\text {sur }}+F_{t}^{\text {bend }}+F_{t}^{\text {elast }}[$ see Eq. (2)] with respect to $r$ with the constraint $N l a^{2} \simeq r^{2} R$ and the condition $\rho=0$ (neutral chain). By inserting this optimum thickness into Eq. (2), one finds $\Delta F_{1} \sim N^{3 / 5}$. The partially collapsed structure is highly unstable due to this large energetic penalty. However, once the torus is charged, even slightly, electrostatic self-energy should be taken into account. Then the torus thickness as a function of $N$ approaches a saturated value for large $N$, which is determined by the balance between the surface and electrostatic self-energy of the torus (note that the optimum torus thickness is controlled by the screening length through the electrostatic self-energy). ${ }^{21,30}$ If the torus thickness does not depend on $N$, then, by inserting $r=$ const. into Eq. (2), one finds $\Delta F_{1} \sim N^{-1}$, indicating that the energetic penalty in the partially collapsed structure becomes less relevant for relatively large $N$. These features are clearly shown in Fig. 6, which plots the profiles of $F_{1}(N)$ around the transition concentrations of guest molecules $\left(\Delta \mu \simeq 0\right.$ ) for $N_{0}=500$, (a) $\alpha=10^{-4}$, and (b) $\alpha=0.2$.

Dividing one torus into two (or more) tori always requires an additional energetic penalty, which comes from the contribution of the nonextensive part of free energy. As we have discussed above, this penalty is small for large $N$ with some finite value of $\alpha$. On the other hand, the possible number of ways that tori and coils can be arranged increases with the number of tori, while the penalty associated with the boundary between the two states is negligible as a consequence of the quasi-one-dimensional nature of the polymer chain. Our theoretical model of a rings-on-a-string structure, thus, resembles one-dimensional Ising model in a sense, i.e., segments in the torus and coil state correspond to up and down spins, respectively. Roughly speaking, changing the concentration of guest molecules corresponds to changing the external field in the Ising model. The essential difference is the presence of long-range interaction along the one- dimensional chain in the collapsed state, which arises from the actual dimensionality of the collapsed morphology of the polymer chain. This fact produces the nontrivial nonextensive part of free energy that depends on the collapsed structure. In the case of a stiff or semiflexible chain with wormlike flexibility in three-dimensional space, the collapsed morphology is a torus, and its size depends on the degree of the surviving charge inside the torus. ${ }^{21}$ Owing to this characteristic, our model exhibits a discrete character on the transition in contrast to the one-dimensional Ising model. Depending on the form of the nonextensive part of free energy, the entropic factor due to the combination of the arrangement of tori and coils in a chain, i.e., combinational entropy, can dominate the energetic penalty with a chain longer than some critical length, which leads to the appearance of a rings-ona-string structure.

The mechanism discussed does not depend on the modeling details. Essential requisites are (i) the discreteness of the collapse transition and (ii) the particular property of a torus, namely, its instability to thicken beyond a certain size. A general nature of our simple homopolymer model suggests the appearance of rings-on-a-string structures not only for DNA molecules but also for other semiflexible polyelectrolytes. In the present model, we ascribe the factor limiting the torus thickness to the charge effect. In this sense, the mechanism is partly analogous to the appearance of pearl-necklace structures of a flexible polyelectrolyte based on the Rayleigh instability. But there may be other situations where the torus thickness is limited by other mechanism. For example, we expect that surfactant molecules, which are sometimes used as condensing agents, may affect such structural property through the packing inside collapsed structures.

As one can expect from Figs. 3 and 4 (also easily guessed from the analogy with one-dimensional Ising model), rings-on-a-string structures exhibit a rather large fluctuation in terms of the number, size of tori, and their position along the chain. Another interesting prediction from the model is the evolution of the number of the collapsed part (ring); after a single ring is formed at the onset of the partial collapse, the number of rings increases with the further addition of the condensing guest molecules. We note that, indeed, recent experiments indicate some of the collapsing behaviors of giant DNA molecules are described by our model, i.e., the torus thickness is limited, and the number of torus increases as the collapse proceeds. ${ }^{16,27}$

\section{CONCLUSION}

We have proposed a plausible mechanism for the occurrence of the coexistence of order and disorder within a single polyelectrolyte chain, i.e., rings-on-a-string structures. We have demonstrated that the nature of the collapse transition critically depends on a property (size scaling) of the torus. If the thickness of the torus is limited by some mechanism, the collapse of a sufficiently long chain is characterized by a sequence of discrete transitions, i.e., swollen coil-(micro) phase separated structure with one torus-two tori-...-fully collapsed state. The presence of finite surviving charge inside the torus has been pointed out as one possible mechanism for 
limiting the torus thickness. ${ }^{21}$ The degree of surviving charge inside a torus $\alpha$ may depend on several factors, such as steric structure and the chemical properties of condensing agents, the concentration of coexisting monovalent and/or other electrolytes, etc. In the present analysis, we assumed that $\alpha$ is a given fixed parameter, and asked how it influences the transition behavior. This is primarily because we sought to make the discussion simple and transparent, which allows us to capture the essence of the mechanism. Another reason lies in the difficulty for incorporating such properties in the model in a consistent manner. It is expected, however, that $\alpha$ may also change to some extent depending on the concentration of guest molecules $c_{g}$. The degree of this change should depend on the experimental systems, but if $\alpha$ decreases rather quickly with the addition of the guest molecules, then, the region for the appearance of "rings-on-a-string" becomes narrower. The analysis of such effects is left for a future problem and further experimental as well as theoretical studies are awaited to make clear the full scenario of the (micro) phase separation on semiflexible polyelectrolytes, both on equilibrium and kinetic aspects.

\section{ACKNOWLEDGMENTS}

The authors thank N. Miyazawa for showing them beautiful AFM images of DNA molecules. One of the authors (T.S.) is grateful to Y. Hayashi and T. Kawakatsu for valuable discussions. The present study was supported by JSPS Research Fellowships for Young Scientists (01263).

\footnotetext{
${ }^{1}$ A. Grosberg and A. Khokhlov, Statistical Physics of Macromolecules (American Institute of Physics, New York, 1994).

${ }^{2}$ C. Post and B. Zimm, Biopolymers 21, 2123 (1982).

${ }^{3}$ V. Bloomfield, Curr. Opin. Struct. Biol. 6, 334 (1996).

${ }^{4}$ K. Yoshikawa, M. Takahashi, V. Vasilevskaya, and A. Khokhlov, Phys. Rev. Lett. 76, 3029 (1996)
}

${ }^{5}$ H. Noguchi and K. Yoshikawa, J. Chem. Phys. 109, 5070 (1998).

${ }^{6}$ Y. Kuznetsov and E. Timoshenko, J. Chem. Phys. 111, 3744 (1999).

${ }^{7}$ T. Sakaue and K. Yoshikawa, J. Chem. Phys. 117, 6323 (2002).

${ }^{8}$ M. Stukan, V. Ivanov, A. Grosberg, W. Paul, and K. Binder, J. Chem. Phys. 118, 3392 (2003).

${ }^{9}$ S. Starodubsev and K. Yoshikawa, J. Phys. Chem. 100, 19702 (1996).

${ }^{10}$ K. Yoshikawa, Y. Yoshikawa, Y. Koyama, and T. Kanbe, J. Am. Chem. Soc. 119, 6473 (1997).

${ }^{11}$ S. Takagi, K. Tsumoto, and K. Yoshikawa, J. Chem. Phys. 114, 6942 (2001).

${ }^{12}$ T. Sakaue, Proceeding in MACRO 2004, 40th International Symposium on Macromolucules, Macro 2004, Paris, France July 4-9, 2004, http:// www.e-polymers.org/paris/, Chap. 4, Session 4.14.

${ }^{13}$ T. Iwaki and K. Yoshikawa, Europhys. Lett. 68, 113 (2004).

${ }^{14}$ Y. Hayashi, Europhys. Lett. 68, 536 (2004).

${ }^{15}$ A. Zinchenko, V. Sergeyev, S. Murata, and K. Yoshikawa, J. Am. Chem. Soc. 125, 4414 (2003).

${ }^{16}$ N. Miyazawa, T. Sakaue, R. Zana, and K. Yoshikawa, J. Chem. Phys. 122, 044902 (2005).

${ }^{17}$ L. Rayleigh, Philos. Mag. 14, 184 (1882).

${ }^{18}$ A. Dobrynin, M. Rubinstein, and S. Obukhov, Macromolecules 29, 2974 (1996).

${ }^{19}$ H. Limback, C. Holm, and K. Kremer, Europhys. Lett. 60, 566 (2002).

${ }^{20}$ D. Baigl, M. Sferrazza, and C. Williams, Europhys. Lett. 62, 110 (2003).

${ }^{21}$ T. Sakaue, J. Chem. Phys. 120, 6299 (2004).

${ }^{22}$ F. Oosawa, Polyelectrolytes (Dekker, New York, 1971).

${ }^{23}$ G. Manning, Q. Rev. Biophys. 11, 179 (1978).

${ }^{24}$ A. Grosberg and A. Zhestkov, J. Biomol. Struct. Dyn. 3, 859 (1986).

${ }^{25}$ J. Ubbink and T. Odijk, Europhys. Lett. 33, 353 (1996).

${ }^{26}$ V. Vasilevskaya, A. Khokhkov, S. Kidoaki, and K. Yoshikawka, Biopolymers 41, 51 (1997).

${ }^{27}$ N. Chen, A. Zinchenko, S. Murata, and K. Yoshikawa, J. Am. Chem. Soc. 127, 10910 (2005)

${ }^{28}$ D. Bratko and K. Dawson, J. Chem. Phys. 99, 5352 (1993).

${ }^{29}$ Our simple homopolymer model is apolar, i.e., no "direction" along the chain. In linear DNA molecules (if not the base pair sequence is symmetrical), the direction gives rise to the discrimination between seemingly the same structures, but with the reversed distribution with respect to the segment states. Inclusion of this effect broadens the region for the rings-on-a-string conformations.

${ }^{30}$ The characteristic length scale of subunits in flexible polyelectrolytes is also controlled by screened electrostatic interactions (Ref. 28). 